\title{
RECOGNITION AND STATISTICAL SIZE DISTRIBUTION OF MICRO- DOMAINS IN TEM-IMAGES OF POLYIMIDE FILMS DURING THEIR PROCESSING
}

\section{SERGEI BRONNIKOV AND TATIANA SUKHANOVA}

Institute of Macromolecular Compounds, Russian Academy of Science, Bolshoi Prospekt 31, 199004 St. Petersburg, Russia

e-mail: bronnik@hq.macro.ru

(Accepted.

\begin{abstract}
The surface morphology of polyamic acid films evolving under heating towards polyimide films is investigated with transmission electron microscopy. Micro-domains observed at the surface of the films are treated statistically and shown to form optimized statistical ensemble(s). The statistical size distribution of the micro-domains is described with the model of reversible aggregation. The distribution parameters, namely the aggregation energy and the mean diameter of micro-domains, are shown to depend on the chemical structure of the polymer and the heating temperature.
\end{abstract}

Keywords: micro-domains, model of reversible aggregation, polyimide films, statistical analysis, TEM-images.

\section{INTRODUCTION}

Thermal stability is an important property of polymeric materials. Aromatic polyimides (PI) show an excellent thermal stability, good mechanical properties, a high chemical resistance, and a low electric conductivity. In this respect, they are widely used in technical applications as thermally stable insulators, surface coverage, fibers, etc. (Bessonov et al., 1987). PI materials are usually produced through a twofold procedure. First, the synthesis of the precursor, a polyamic acid (PAA) dissolved in a polar solvents; second, the conversion (imidization) of PAA to PI. The latter step may be expressed as:
The morphology of PAA films all along their conversion to PI has been thoroughly studied with transmission electron microscopy (TEM) (Bessonov et al., 1987). Yet, to the authors' knowledge, the statistical analysis of micro-domain ensembles has still not been carried out. The latter issue is addressed in this paper towards revealing new aspects of imidization.

The goals of the communication are: i) the identification and statistical analysis of micro-domain ensembles at the surface of PAA films during their conversion to PI under heating, ii) the subsequent description of the micro-domain statistical size distributions with the model of reversible aggregation, and iii) the analysis of the distribution parameters as a function of the heating temperature.

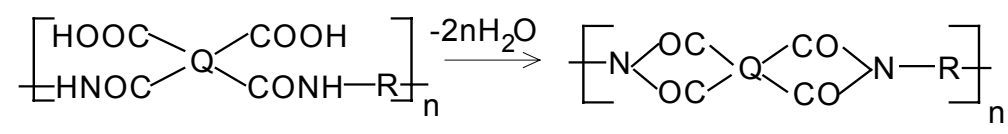

PAA

PI

where $\mathrm{Q}$ and $\mathrm{R}$ are radicals.

Imidization has been investigated with different methods. It is known to happen along with such phenomena as evaporation of the residual solvent, and crystallisation in some cases. Because imidization and its accompanying processes influence the final structure and the properties of PI, they should be carefully analyzed towards controlling the final properties of PI materials.

\section{EXPERIMENTAL PROCEDURE}

The PAA films under investigation were prepared from i) 3,3',4,4'-tetra carboxylic dianhydride and $p$ phenylene diamine (PAA DPP), and ii) pyromellyte dianhydride and 2,7- fluorene diamine (PAA PMF).

Films with a thickness of $40 \pm 5 \mu \mathrm{m}$ were cast from 12 wt \% PAA solutions in $N, N^{\prime}$-dimethyl formamide 
onto a glass substrate, and dried in air for two days. Then the films were heated up to a chosen temperature (maximum: $420{ }^{\circ} \mathrm{C}$ ) at a rate of $2 \% \mathrm{~min}$ in an inert medium (nitrogen) to produce PI through the following reactions: in the TEM-images of replica. The thicker carbon layer of the replicas was prepared by high vacuum evaporation from a carbon arc on the surfaces. The $\mathrm{Pt} / \mathrm{C}$ replicas were then exfoliated from the etched surface of films with an aqueous solution

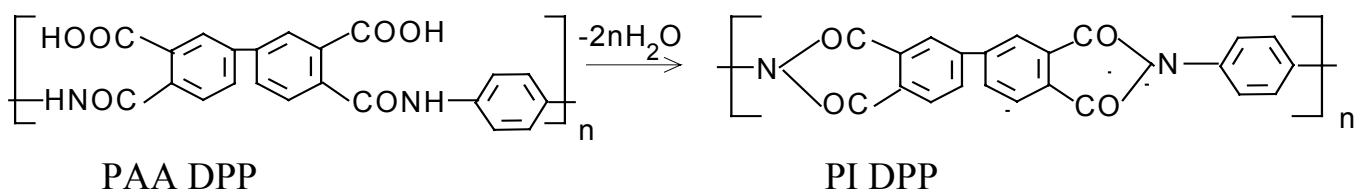

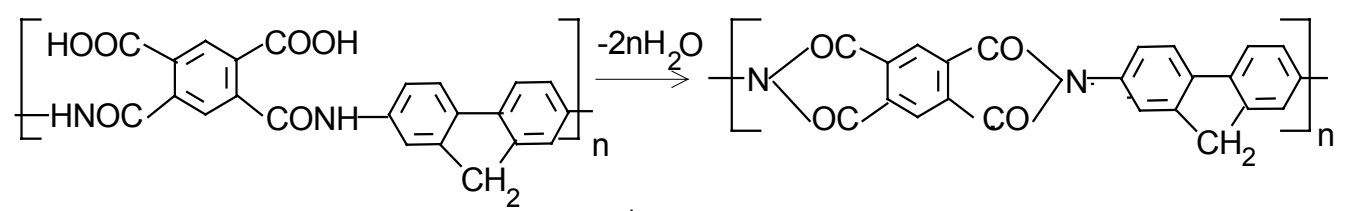

PAA PMF

Once removed from the substrate, the films showed many defects, probably due to mechanic distortion. Film surfaces turned out to be also polluted, so that the super-molecular (micro-domain) structure could not be identified in the TEM-images. To access by TEM the micro-domain structure of interest, the defective components had to be removed. In this respect, the surface of the samples was etched by submitting it to high frequency discharge plasma in the way and with a device similar to Neppert et al., (1983). The etching time varied from 15 min (PAA films) to $60 \mathrm{~min}$ (PI films heated above $300^{\circ} \mathrm{C}$ ). During exposure, the surface temperature did not exceed $50^{\circ} \mathrm{C}$. In such operational conditions, imidization could not be (re)-activated and sputtering was accordingly not observed. Of course, one must accept that the resulting micro-domain structure may not be exactly identical to the original one. Yet, even in this case, any deviations should be small as ensured by the very soft conditions of the treatment (Neppert et al., 1983).

As far as the etched films were too thick to be investigated directly with TEM, they were immediately replicated, after shadowing first with a very thin platinum $(\mathrm{Pt})$ layer at an angle of $30^{\circ}$. The diameter of platinum grains being smaller than $1 \mathrm{~nm}$, we were able to identify even very small micro-domain of polyacrylic acid (PAA) and cut away after drying using a razor blade. The replicas were placed in distilled water during about $2 \mathrm{~h}$ to remove the PAA Finally, $\mathrm{Pt} / \mathrm{C}$ replicas in the form of $30-40 \mathrm{~nm}$ thick films were ultimately adsorbed on the copper grids, and were afterwards investigated with a Tesla BS-500 transmission electron microscope at magnifications $2 \times 10^{4}-4 \times 10^{4}$. One example TEM-image of the surface of an etched and replicated sample is provided in Fig. 1a. The surface consists of microdomains (super molecular entities) of varying size and creating a positive relief at the film surface.

For quantitative analysis of the size of microdomains, the TEM-images were segmented and treated with the software Image Tool 3.0 (Health Science Center, University of Texas). In the example binary Fig. 1b, the micro-domains are expressed in black. The segmented micro-domains were systematically then subjected to area measurements. Subsequently their statistical size distribution was drawn. The number of micro-domains analyzed per image ranges from 600 to 1400 (ensuring statistical representativity). As discussed further on, the statistical analysis revealed that the observed surface micro-domains form statistical ensembles that can be described with the model of reversible aggregation. 
a)

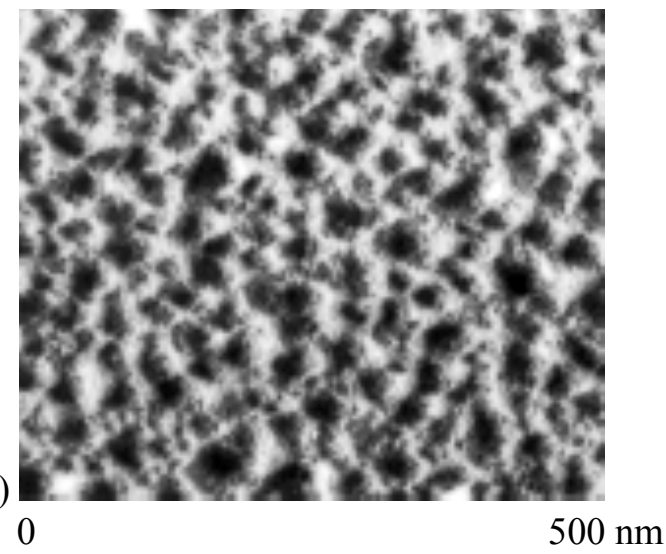

b)

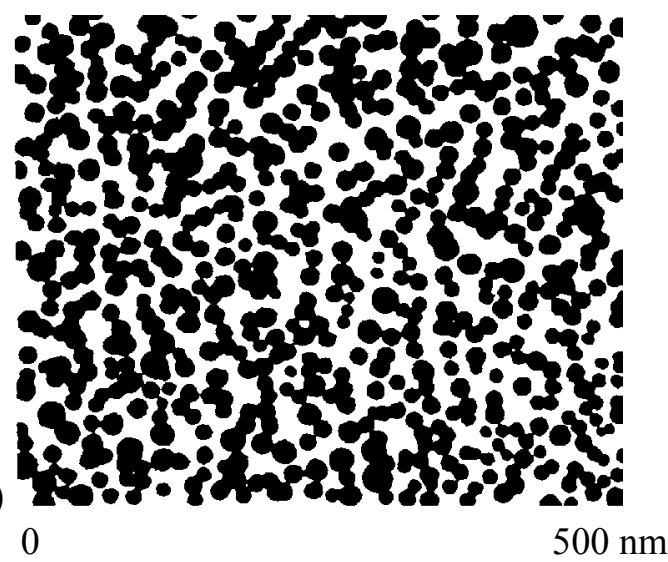

Fig. 1. (a) grey level TEM-image (Sample: PAA DPP) showing micro-domains distributed at the film surface. Here the protocol discussed in section Experimental Procedure enhanced the (height-) contrast between the micro-domains of interest (dark greys) and their complement (inter-domain regions) (b) Binarized counterpart for digital analysis where the circular micro-domains in Fig. 1a (dark grey levels) are expressed in black.

\section{MODEL}

Micro-domains observed at the surface of PAA films all along their conversion (under heating) to PI were analysed with the model of reversible aggregation (Kilian et al., 1997; 2001; Köpf and Kilian, 1999). This model describes stationary ensemble properties whose characteristics are evaluated. The basic assumption of the model is that stationary structures are generated by the aggregation of identical units in metastable clusters called aggregates. Aggregates behave as a heterogeneous phase, large enough so as to allow the application of thermodynamics. Aggregates are assumed to comprise $y_{a}$ identical units and to vary largely in size. The number of unit-to-unit contacts $y$ in an aggregate is defined as $y=y_{a}-1$. The aggregation energy $\Delta u[\mathrm{~J}]$ should therefore grow proportional to $y$ :

$$
\Delta u=y \Delta u_{0},
$$

where $\Delta u_{0}[\mathrm{~J}]$, the standard aggregation energy per elementary identical unit, is supposed to be constant in all aggregates, independently of their size. $\Delta u$ being commensurable with $k T\left(k\left[\mathrm{~J} \cdot \mathrm{K}^{-1}\right]\right.$ is the Boltzmann constant and $T[\mathrm{~K}]$ is the absolute temperature), the formation and the decomposition (reversible aggregation) of metastable aggregates are thermally activated. By analogy with many simultaneous and reversible linear chemical reactions, the thermodynamic interpretation of the reaction constant indicates that aggregation is controlled by the standard aggregation energy $\Delta u_{0}$. The dimensionless fraction number $n(y)$ of aggregates with $y$ unit-to-unit contacts can be expressed as (Kilian et al., 1997, 2001; Köpf and Kilian, 1999):

$$
n(y)=a y^{p} \exp \left(-\frac{y \Delta u_{0}}{k T}\right)
$$

where $a$ is a normalizing factor, and $p$ defines the class of the aggregate ensemble with respect to the dimension $D$ of the space where the aggregation occurs $(D=p+1)$. Under our hypothesis, the size (area) of an aggregate and the number of unit-to-unit contacts $y$ are intimately related (proportional). For this reason, $y$ will represent from now on the area of an aggregate and will be expressed in $\left[\mathrm{nm}^{2}\right] ; \Delta u_{0}$ will carry accordingly further on the dimension $\left[\mathrm{J}_{\mathrm{nmm}}{ }^{-2}\right]$. Multiplying the fraction number (Eq. 2) by $y\left[\mathrm{~nm}^{2}\right]$ yields the size distribution of the aggregates $h(y)$ :

$h\left(y-y_{0}\right)=a\left(y-y_{0}\right)^{p+1} \exp \left(-\frac{\left(y-y_{0}\right) \Delta u_{0}}{k T}\right)$,

where $a$, a normalizing factor, is now expressed in $\left[\mathrm{nm}^{-4}\right]$. Note that the minimum (embryonic) aggregate size $y_{0}\left[\mathrm{~nm}^{2}\right]$ has been introduced. Eq. 3 may be interpreted as the probability density of a thermodynamic phase, i.e., a canonical Gibbs distribution (Lavenda, 1991).

Over the last few years, the current model has been successfully applied to the description of ensemble structural entities in polymers showing different chemical structures (Kilian et al., 1997, 2001; Köpf and Kilian, 1999; Bronnikov and Sukhanova, 2001; Bronnikov et al., 2002), nuclei in liquid crystals during their transition from a isotropic liquid to a nematic phase (Zuev and Bronnikov, 2002), carbon black particles in natural rubber (Köpf and Kilian, 1999; Kilian et al., 2001), bacteria, and yeast (Kilian et al., 2001). 


\section{EXPERIMENTAL DATA AND THEIR INTERPRETATION}

Fig. 2 shows a set of representative micrographs of the $\mathrm{C} / \mathrm{Pt}$ replicas of the etched surface of PAA DPP and PI DPP films heated up to different temperatures (see captions in Fig.2). The surface of the films is seen to be formed by micro-domains of nearly spherical shape whose size and packing depend on the final heating temperature.

a)

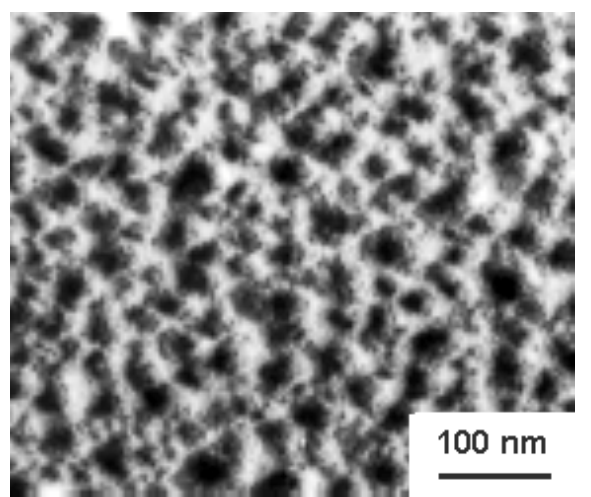

b)

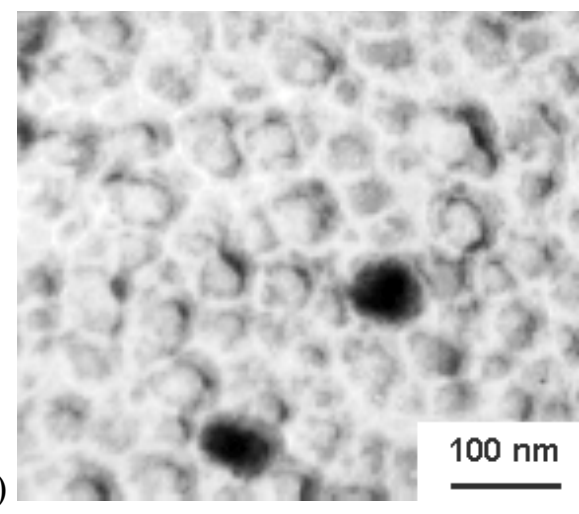

c)

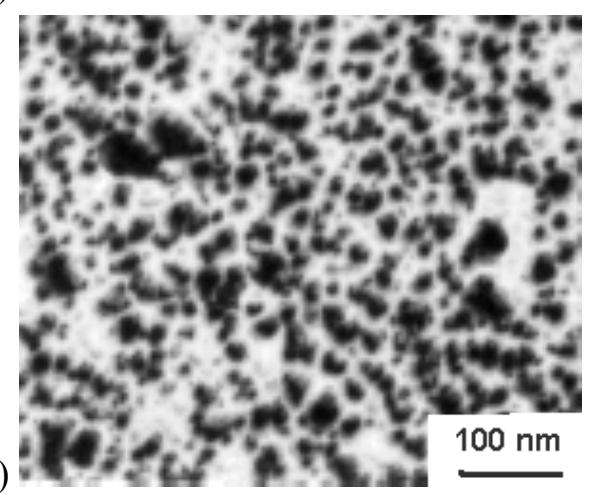

Fig. 2. TEM-micrographs of the surface of (a) PAA DPP and PI DPP samples heated up to (b) 200 and (c) $420^{\circ} \mathrm{C}$.

Fig. 3 displays the statistical distribution of the micro-domain diameters of the samples in Fig. 2; micro-domain diameter $d[\mathrm{~nm}]$ (in histograms) and their size $y\left[\mathrm{~nm}^{2}\right]$ (Eq. 3) are related according to $d=y^{1 / 2}$. The histograms in Fig. 3 evidence a single statistical ensemble of micro-domains.

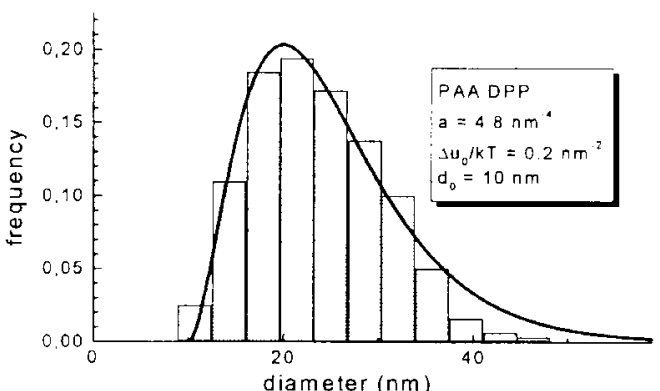

a)

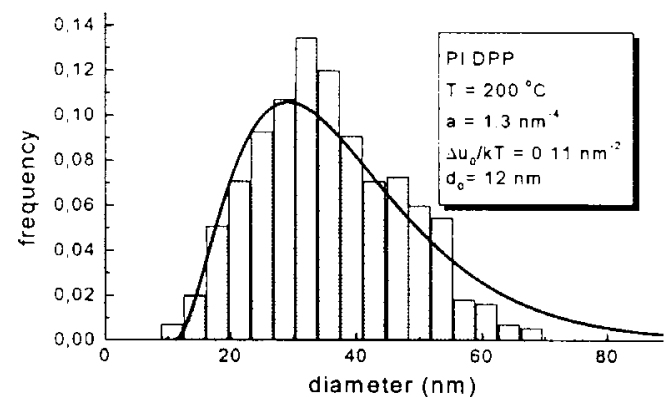

b)

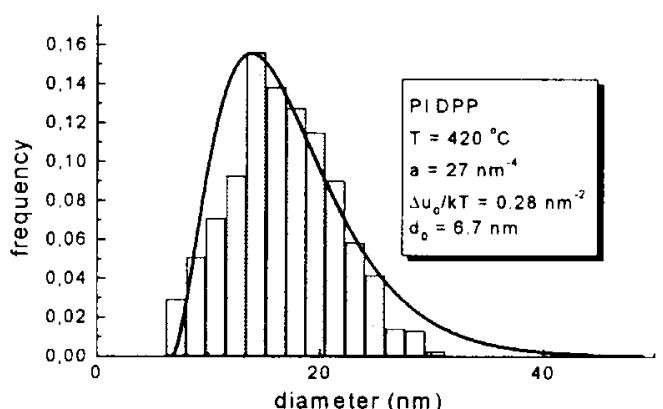

c)

Fig. 3. Statistic distribution of the micro-domain diameter at the surface of (a) PAA DPP and PI DPP samples heated up to (b) 200 and (c) $420^{\circ} \mathrm{C}$.

When applying the model of reversible aggregation in the form of Eq. 3, micro-domains were treated as 'identical units' while their statistical ensemble as an 'aggregate'.

Solid lines in Fig. 3 show the adjustment (curve fitting) of Eq. 3 to the experimental histograms for PAA DPP and PI DPP heated up to different temperatures. Here, $a, \Delta u_{0} / k T$, and $d_{0}=y_{0}{ }^{1 / 2}$ are tuned (adjusted) parameters (their values are listed in Fig. 3 ) whereas $p$ is a fixed parameter $(p=1$ in agreement with the $2 \mathrm{D}$ version of the model of reversible aggregation used herein (Kilian et al., 2001)).

As shown in Fig. 3, the statistical distribution of the micro-domain diameters of the patterns investigated may be successfully described with Eq. 3 for the samples depicted in Fig. 2. This evidences that optimized micro-domain ensembles arise consistently (at every heating temperature) during thermal conversion. 
TEM-images of the surface of the PAA PMF films during their conversion to PI PMF are presented in Fig. 4. The obtained statistical distributions for their micro-domain diameters are shown in Fig. 5.

a)

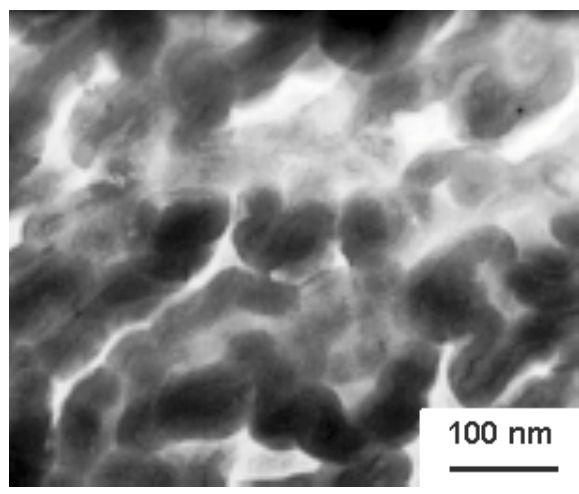

b)

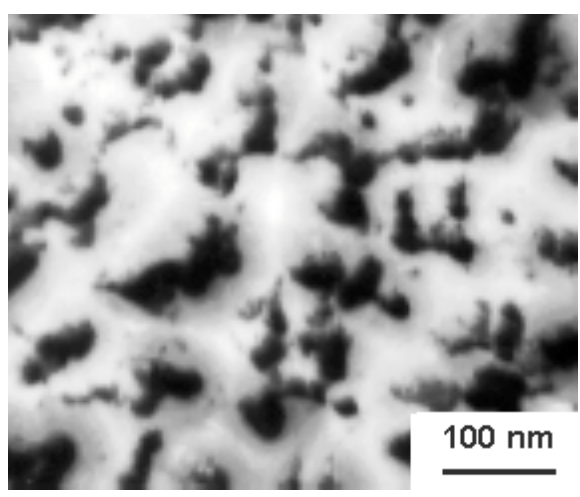

c)

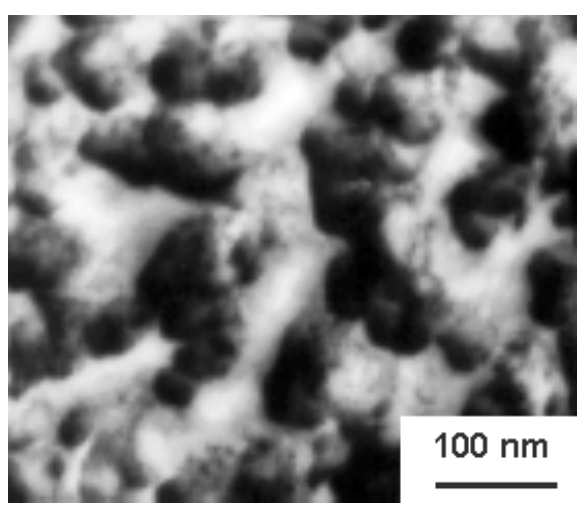

d)

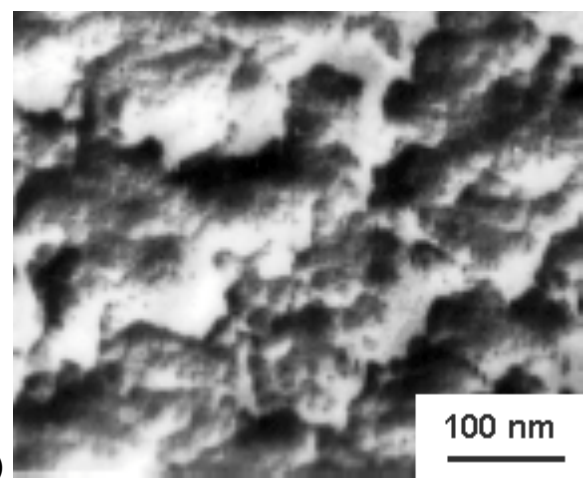

Fig. 4. TEM- micrographs of the surface of (a) PAA $P M F$ and PI PMF samples heated up to (b) 125, (c) 250 , and $(d) 400{ }^{\circ} \mathrm{C}$.

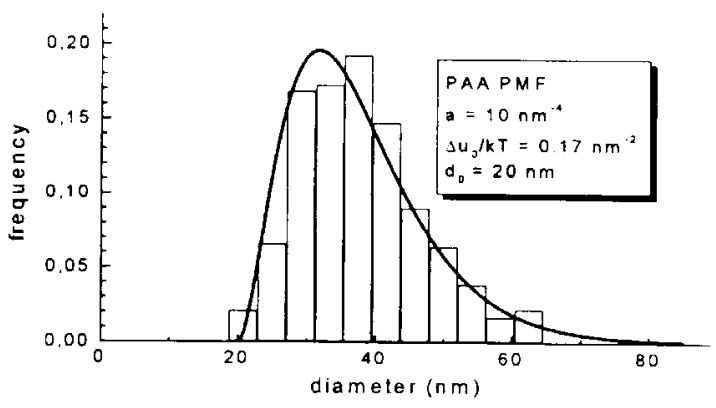

a)

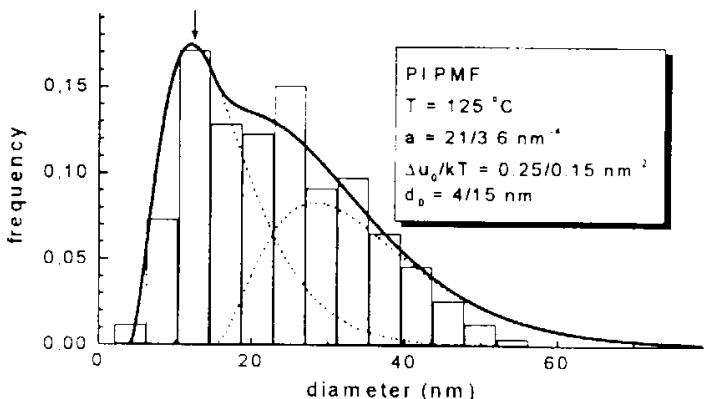

b)

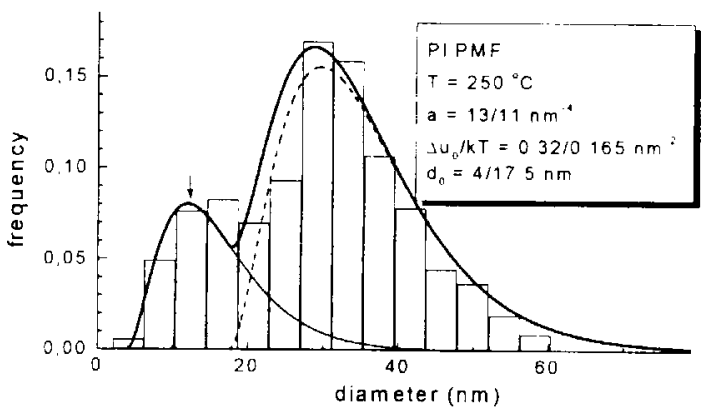

c)

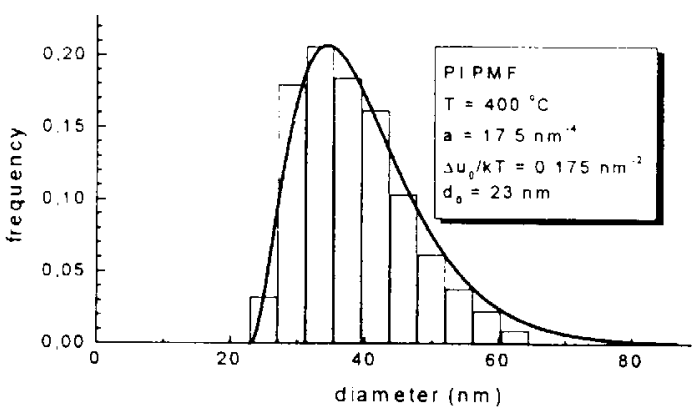

d)

Fig. 5. Statistic distribution of the micro-domain diameter at the surface of (a) PAA PMF and PI PMF samples heated up to (b) 125 , (c) 250 and (d) $400{ }^{\circ} \mathrm{C}$.

As far as PAA PMF and PI PMF samples are concerned, two (a and d) of the experimental histograms presented in Fig. 5 could be interpreted with Eq. 3 (solid lines in Figs 5a and 5d). However, the generalized form of the model of reversible aggregation expressed by Eq.4 was used (with $n=2$ ) to interpret the histograms of the PI PMF samples heated up to 125 and $250^{\circ} \mathrm{C}$ (Figs. 5b-c). 


$$
\begin{aligned}
& h\left(y-y_{0}\right)= \\
& \quad \sum_{i}^{i=n} a_{i}\left(y_{i}-y_{0 i}\right)^{p+1} \exp \left(-\frac{\left(y_{i}-y_{0 i}\right) \Delta u_{0 i}}{k T}\right),
\end{aligned}
$$

where $n$ is the number of statistical ensembles.

Such an interpretation based on Eq. $4(n=2)$ shows that the micro-domains form two overlapping statistical ensembles. In case of bimodal distribution (Fig. 5b-c), the dotted lines show the distribution of each of the two modes. In the TEM-images (Fig. 4b-c) one cannot observed directly the two ensembles whose existence is only revealed by the statistical analysis.

The statistical distribution of the micro-domain diameters may be characterized by the mean size $\langle y\rangle$ (normalized mathematical expectation):

$$
<y>=y_{0}+\frac{\int_{0}^{\infty}\left(y-y_{0}\right) h\left(y-y_{0}\right) d y}{\int_{0}^{\infty} h\left(y-y_{0}\right) d y} .
$$

For any modes, described by Eq. 4, Eq. 5 may be expressed as:

$$
<y>=y_{0}+\frac{(p+2) k T}{\Delta u_{0}} .
$$

The mean diameter of micro-domains is accordingly defined as $\langle d\rangle=\langle y\rangle^{1 / 2}$.

The distribution parameters $\Delta u_{0} / k T$ and $<d>$ are presented as a function of the final heating temperature in Fig. 6 (PAA DPP and PI DPP) and in Fig. 7 (PAA PMF and PI PMF).

\section{DISCUSSION}

The analysis of $\Delta u_{0} / k T$ and $\langle d>v s$. final heating temperature (T) (Figs. 6-7) suggests that above $20^{\circ} \mathrm{C}$, the aggregation energy decreases with $T$ towards a minimum between $100^{\circ} \mathrm{C}$ and $200^{\circ} \mathrm{C}$. At higher temperatures, $\Delta u_{0} / k T$ increases and achieves an almost constant value at about $350^{\circ} \mathrm{C} .<d>$ exhibits a mirror (i.e. inverse) evolution with $T$. Our results remain compatible with the achievement of extrema values for both $\Delta u_{0} / k T$ and $\langle d\rangle$ at $T \cong 170{ }^{\circ} \mathrm{C}$, a temperature often referred to as specific for the process of imidization. For example, Bessonov et al., (1987) reported that around this specific temperature $\left(170^{\circ} \mathrm{C}\right)$ an intensive chemical conversion occurs along with a decrease of the molecular mass of polymer. Such conditions are very favorable for the arrival of the imide structure. For higher temperature (above $200^{\circ} \mathrm{C}$ ), the increase of $\Delta u_{0} / k T$ is associated to the restitution of the molecular mass of polymer (Bessonov et al., 1987).

a)
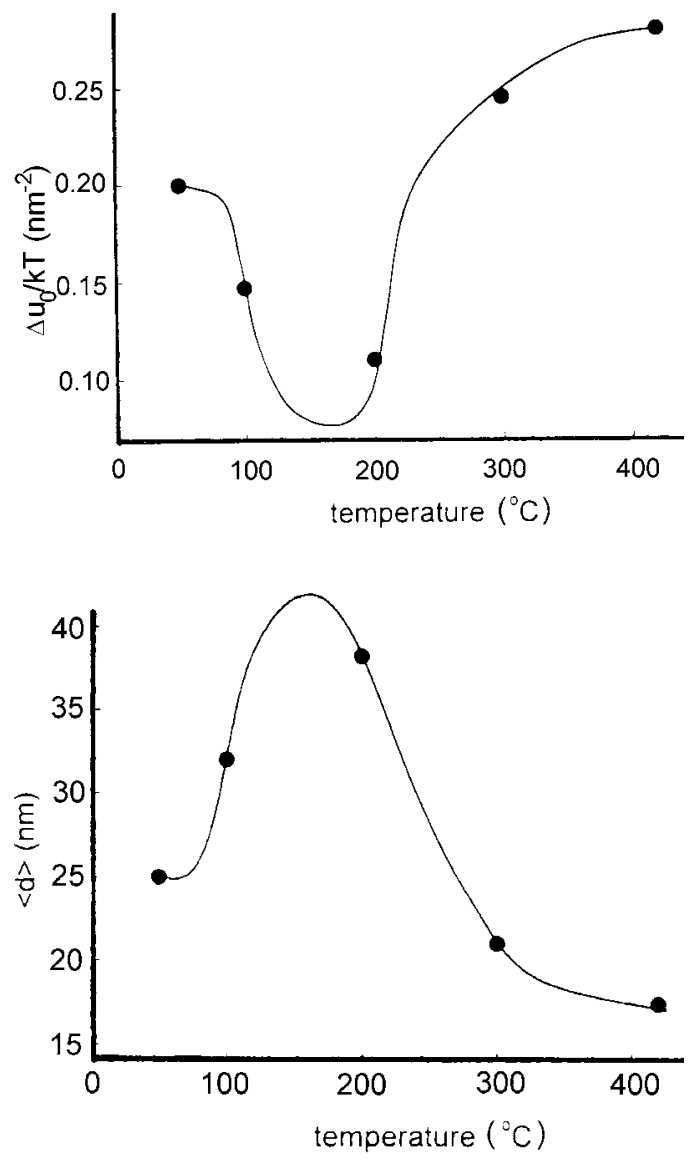

b)

Fig. 6. Parameters of the size distribution, (a) $\Delta u_{0} / k T$ and $(b)\langle d\rangle$, against the final heating temperature. Samples: PAA DPP and PI DPP.

Herein, an interesting observation for PI PMF samples is the existence of an additional ensemble of at temperatures $T=125^{\circ} \mathrm{C}$ and $T=250^{\circ} \mathrm{C}$ identified in Figs. 5b-c with arrows. At $T=125{ }^{\circ} \mathrm{C}$, the aggregation energy is higher for the additional ensemble $\left(\Delta u_{02} / k T\right)$ than for the basic ensemble $\left(\Delta u_{01} / k T\right)$. Therefore, the mean diameter of the microdomains within the additional ensemble is smaller than within the basic ensemble. The extremum of the diameter distribution is about twice higher for the additional ensemble than for the basic ensemble; in other words, the number of micro-domains is larger within the additional ensemble than within the basic one. At $T=250^{\circ} \mathrm{C}$, this trend is reversed, the number of micro-domains within the basic ensemble being larger than within the additional one. Finally, within 
the range $350-400^{\circ} \mathrm{C}, \Delta u_{01} / k T$ reaches a constant value. As far as the additional ensemble vanishes in this temperature range, we may suppose that $\Delta u_{02} / k T$ becomes infinitely large and that $\left\langle d_{2}\right\rangle$ becomes accordingly infinitely small. Of course, such results are based on a limited number of experimental points, particularly for the additional ensemble.

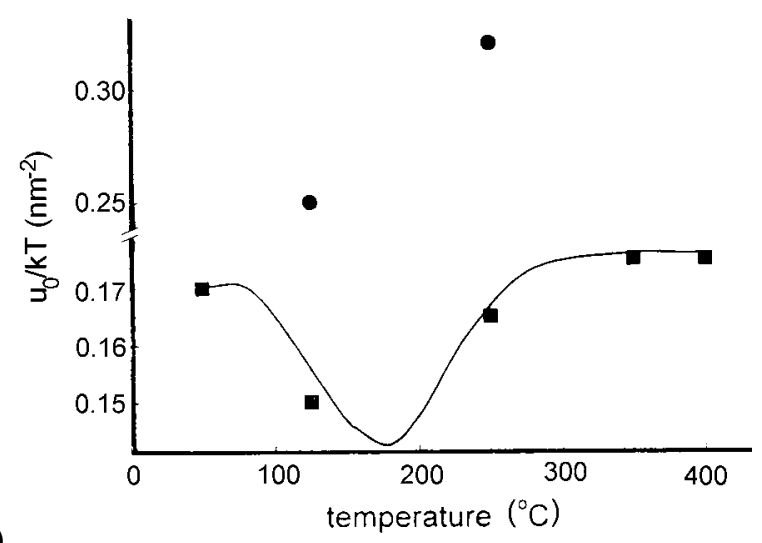

a)

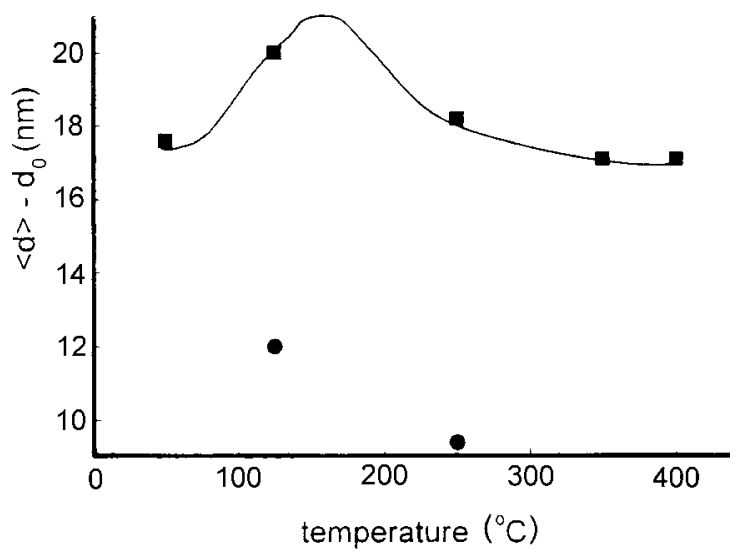

b)

Fig. 7. Parameters of the size distribution, (a) $\Delta u_{0} / k T$ and (b) $\langle d\rangle-d_{0}$, against the final heating temperature. Basic and additional statistical ensembles are denoted as and - Samples: PAA PMF and PI PMF.

The appearance of a second ensemble of microdomains in PAA PMF under heating may be explained by the sample specific chemical structure. Within the range $125-150^{\circ} \mathrm{C}$, PAA PMF macromolecules are destroyed, and oligomers containing fluorene fragments appear (Bell, 1976). This leads to the creation of a new cured structure, whose micro-domains may form the additional statistical ensemble (Figs. 5b-c). At the highest temperatures, this cured structure is supposed to be destroyed by heating so that only the final imide structure is revealed.

\section{CONCLUSIONS}

Micro-domains of different size and shape have been recognized in the TEM-images of the surface of the PAA films during their thermal conversion to PI. Micro-domains have been shown to form statistical ensemble(s) whose size distributions were successfully described with the model of reversible aggregation. The evolution with heating temperature of such distribution parameters as the aggregation energy and micro-domain mean diameter was analyzed, revealing for the investigated samples similar trends. Yet some peculiarities were acknowledged due to the different chemical structure of the studied polymers. Particularly, for some heating temperatures, microdomains related to different intermediate chemical structures of the PAA PMF samples seem to form two overlapping statistical ensembles as opposed to one in all other examples. It is our hope that the current approach can be suitable for to the analysis of other solid-state chemical reactions.

\section{REFERENCES}

Bell VL (1976). Polyimide structure-property relationships. 1. Polymer from fluorene-derived diamines. J Polym Sci: Chem Ed 14:225-35.

Bessonov MI, Koton MM, Kudryavtsev VV, Laius LA (1987). Polyimides - thermally stable polymers. New York: Plenum Press.

Bronnikov S, Sukhanova T (2001). Statistical aspect of the polyimide films morphology. Image Anal Stereol 20(Suppl 1):133-8.

Bronnikov S, Sukhanova T, Laius L (2002). Surface morphology of polyamic acid and polyimide films prepared from 3,3',4,4'-biphenyltetracarboxylic dianhydride and p-phenylenediamine. Polymer Sci A 44:576-80.

Kilian HG, Metzler R, Zink B (1997). Aggregate model of liquids. J Chem Phys 107:8697-714.

Kilian HG, Kopf M, Vettegren VI (2001). Model of reversible aggregation: Universal features of fluctuating ensembles. Progr Colloid Polym Sci 117:172-81.

Köpf M, Kilian HG (1999). Relaxation in the glass transition regime interpreted in the terms of the aggregate model. Acta Polym 50:109-21.

Lavenda B L (1991). Statistical Physics: A Probabilistic Approach. New York: J. Wiley \& Sons.

Neppert B, Heise B, Kilian HG (1983). Ion etching of polymers. Colloid Polym Sci 261:577-84.

Zuev V, Bronnikov S (2002). Stationary statistical size distribution of nematic droplets in the course of the isotropic liquid-nematic phase transition. Liquid Crystals 29:1279-81. 